\title{
Translation and psychometric properties of the "ethical conflict in nursing questionnaire: critical care version" in Iran
}

Fatemeh Motaharifar ${ }^{1}$, Foroozan Atashzadeh-Shoorideh², Amir Hosein Pishgooie ${ }^{3}$, Anna Falcó-Pegueroles ${ }^{4}$

${ }^{1}$ M.Sc. Management Nursing Student, Department of Nursing Management, School of Nursing \& Midwifery, Shahid Beheshti University of Medical Sciences, Tehran, Iran

${ }_{2}^{2}$ Ph.D. in Nursing, Assistant Professor, Department of Nursing Management, School of Nursing \& Midwifery, Shahid Beheshti University of Medical Sciences, Tehran, Iran

${ }^{3}$ Assistant Professor, Ph.D., M.Sc., B.Sc., RN., Faculty of Nursing, AJA University of Medical Sciences, Tehran, Iran

${ }^{4}$ Ph.D. in Nursing, MHSc., RN, Department of Fundamental Care and Medical-Surgical Nursing, School of Nursing., Faculty of Medicine and Health Sciences. University of Barcelona, Barcelona, Spain

\section{Type of article: Original}

\begin{abstract}
Introduction: Ethical conflict is one of ICU nurses' main problems, which rise for several reasons that must be measured. Unfortunately, there is no native instrument for measuring ethical conflicts for ICU nurses in Iran. One of the more suitable and new tools for the measurement of ethical conflict is called the "Ethical Conflict in Nursing Questionnaire: Critical Care Version (ECCNQ-CCV)." This study was aimed to translate and investigate psychometric properties of the ECCNQ-CCV in Iranian nurses.

Methods: In this methodological study, after translation and cultural adaptation, face validity, content validity, and construct validity were assessed. Then, internal consistency and stability were measured for reliability. Two software programs (SPSS version 20 and AMOS) were used for data analysis.

Result: Face and content validities were acceptable. Confirmatory factor analysis was not fitted. Thus, exploratory factor analysis was done, which showed five factors. However, some of the scenarios in a factor were not compatible with each other, and choosing the fit name for factors was not possible. Thus, all the scenarios were put into one factor, which has been proposed by the developer in the original version. The alpha Cronbach was 0.92 for the total scale.

Conclusions: Findings show that the one factor Persian version of the ECNQ-CCV has acceptable psychometric properties. It can be used to evaluate ethical conflicts in Iranian ICU nurses.

Keywords: Ethical conflict, Psychometric properties, Nursing
\end{abstract}

\section{Introduction}

All nurses are susceptible to ethical conflict (1), which occurs when there are differences between individuals' values and organizations' values, typically manifested through the actions of organization's administrators (2). As a result, nurses will understand emotional distress, which may lead to harmful outcomes for the nurse and the organization $(1,3,4)$. Consequently, nurses will experience emotional distress, which may result in adverse outcomes for the nurse and the organization (1). Ethical conflict is natural for health professions, due to inevitable staff relationships and the ethical responsibilities in patient caring (2). Studies about nurses' ethical conflicts have begun in the two last decades $(1,3-5)$. But there is a little known about nurses' ethical conflicts and the typically resulting unfavorable consequences (1). Nurses need to know what is an ethical conflict and what are the sources of conflict $(6,7)$, which is necessary to reduce and prevent exposition because ethical conflict represents a barrier

\section{Corresponding author:}

Assistant Professor Dr. Foroozan Atashzadeh-Shoorideh, Department of Nursing Management, School of Nursing \& Midwifery, Shahid Beheshti University of Medical Sciences, Tehran, Iran.

Tel: +98.2188655366, Fax: +98.2188202521, Email: f_atashzadeh@sbmu.ac.ir

Received: October 19, 2016, Accepted: January 15, 2017, Published: February 2017

iThenticate screening: December 26, 2016, English editing: February 01, 2017, Quality control: February 06, 2017

(C) 2017 The Authors. This is an open access article under the terms of the Creative Commons Attribution-NonCommercialNoDerivs License, which permits use and distribution in any medium, provided the original work is properly cited, the use is non-commercial and no modifications or adaptations are made. 
against providing high quality of nursing care to patients and their families (6-10). For this reason, it is necessary to measure ethical conflict with a valid and reliable tool. After recognizing an ethical conflict level, recommended strategies to reduce or decrease the exposure to it must be presented (7, 11-13). Obviously, an ethical conflict is a problem with multiple negative consequences that may threaten quality of nursing care and ethical integrity in the nursing profession. Thus, it is needed for measuring the level of nurses' ethical conflicts, particularly in the ICU (3), where nurses are typically faced with moral conflicts (14). The ICU is where a multi-professional team works together to care for critically ill patients (15). ICU workers are confronted with workloads, communication, and endof-life care, relationships with other clinical staff, emotional demands of caring, and shift working (16). Conflicts are common in the ICU and cause high-level stress to all those involved. There are significant factors that lead to conflicts in the ICU (17). Thus there are three main resources for perceived ethical conflicts by nurses: the first is close communication with patients and their families; the second is providing special treatments; the last is the characteristics of the ICU workplace in which the clinical team works (2). Some of research revealed ethical conflicts in ICU nurses. Wlodarczyk and Lazarewicz found a positive relationship between professional burnout and frequency of ethical conflicts among nurses in Poland (18). In Spain, the findings showed high levels of exposure to ethical conflicts (8). One study revealed that $71.6 \%$ of ICU professionals experienced various kinds of conflicts, such as workload, inappropiate communication, and end-of-life caring, which are typically ethical in nature (16). The major adverse outcomes of conflict are individual effects, interpersonal relationships, and organizational effects. Individual effects are job stress, job dissatisfaction, and absenteeism, leaving the profession, increased discontent, psychosomatic complaints, and negative emotions. Interpersonal relationship effects are negative perception of others, animosity, and avoidance. Organizational effects are diminished coordination and collaboration and reduced efficiency (19). Therefore, more research is needed for identifying ethical conflict levels. In the other words, it seems vital to exert changes into the profession in order to reduce ethical conflict in critical care nurses; in addition, what is more important is measuring the effectiveness of these changes; to do this, a reliable validated scale is needed. In the last 15 years, several authors have created instruments to measure the ethical conflict in health sciences professions, e.g., the moral distress scale by Mary Corley (20), the moral distress questionnaire by Arnetz et al. (21), the stress of conscience questionnaire by Anne-Louise Glasberg (22), and the ICU moral distress by Shoorideh et al. (13). These instruments possess good psychometric properties. However, the questionnaires are based on the measure of moral distress and do not consider the other types of ethical conflict that Jameton identified (23). One of the appropriate and new tools for the measurement of ethical conflict is the "Ethical Conflict in Nursing Questionnaire: Critical Care Version" $(2,3,8)$ by Falcó-Pegueroles, who developed it on the context of Spanish critical care nurses and considered four types of ethical conflict and two moral states. ECNQ-CCV is a valid and reliable instrument, Cronbach's alpha $=0.882$, with 19 items that explain a critical care scenario in which nurses might experience an ethical conflict. This questionnaire measures ethical conflict exposure through three questions for each scenario, frequency of ethical conflict exposure, degree of ethical conflict perceived, and type of ethical conflict experienced. The catogories rating of "frequency of ethical conflict exposure" is never, almost never, at least once a year, at least once every six months, at least once a month, and at least once a week. The rating of "degree of ethical conflict perceived" is based on no problem at all, mildly problematic, fairly problematic, considerably problematic, and highly problematic. Also, the catogory rating for "type of ethical conflict experienced" is based on moral uncertainty, moral dilemma, moral distress, and moral outrage. Moral well-being and moral indifference referred to no ethical conflict. The range of score for each item is from 0 (zero frequencyzero intensity) to 25 (high frequency - high intensity) (which is the related the categories [" 5 - at least once a week" $\times$ "5 - highly problematic"]). The level of ethical conflict is measured by frequency conflict multiplied by intensity conflict, which is called the "variable index of exposure to ethical conflict" (IEEC). The range of the IEEC is 0 to 475 , the upper limit being the product of $19 \times 25(19=$ the number of scenarios exit in the ECNQ-CCV $\times 25=$ maximum value of the IEEC for each situation) $(2,8)$. However, in spite of the importance of the concept of ethical conflict in critical care nurses, no valid and reliable instrument exists in this regard in the Persian language. Therefore, the aim of this study was to translate to Persian language and to investigate psychometric properties of ECNQ-CCV in Iranian critical care nurses.

\section{Material and Methods \\ 2.1. Design and setting}

The present study is based on methodological research (24) and was done in two phases. The first phase was translation and cultural adaptation of the English version of ECNQ-CCV. The second phase was the validation (face validity, content validity, construct validity, and reliability) of a Persian language version of ECNQ-CCV. Samples in the translation phase were four fluent translators to Persian and English languages for forward and backward translation. In the psychometric phase, the study samples were 15 critical care nurses for assessing face validity; 15 
specialists in psychology and ethics for assessing content validity; 190 nurses in the critical care units of Modarres, Imam Hussein, Taleghani, Shohada Tajrish, Luqman and, Masih Daneshvari hospitals in Tehran for assessing construct validity; and 30 critical care nurses for assessing reliability. The inclusion criteria in construct validity were willingness to participate in research, at least one year of experience in critical care unit, at least a bachelor's degree in nursing, and no history of mental disorders.

\subsection{Phase I: Translation and cultural adaptation}

At first, the instrument was translated and culturally adapted. This translation was performed based on the approach proposed by Polit and Yang (25).

\subsubsection{Forward translation}

Two native translators fluent in English and Persian (expert committee) individually translated the English version of the instrument into Persian. The translators were asked to rate the difficult to translate each item on a scale from 0 (not at all difficult) to 100 (extremely difficult).

\subsubsection{Combination of early translations (synthesis)}

The various versions of the translations were reviewed in face-to-face meetings, item by item, with the aim of achieving consensus regarding the best possible translation for each Polit and Yang (25). The expert committee compared the two translations, and the final version was prepared after applying a few changes.

\subsubsection{Back-translation}

The final translation of ECNQ-CCV was translated back into English by two different translators, whose mother tongues are English and are fluent in both languages of English and Persian.

\subsubsection{Reconciliation}

In this step, a final reconciliation was done with a committee of experts and the original developer of the instrument. The goal of this step is to consolidate all the information from the translations, to assess the extent to which meaning of the words and conceptual equivalence has been achieved, and to develop a "pre-final" of the Persian version ECNQ-CCV for further testing.

\subsubsection{Pre-testing and cognitive interviewing}

In order to exam the tentative final version, 15 nurses (these nurses did not participate in the final study) were randomly selected from critical care units. The nurses sent us their opinions about difficulty, irrelevancy, and ambiguity about each item (qualitative face validity).

\subsubsection{Final version}

After combining some minor revisions, the final Persian of the instrument was made.

\subsection{Phase II: Validation of Persian version of ECNQ-CCV}

To confirm the face validity by quantitative method (item impact method), the final instrument was given to 15 ICU nurses (who did not participate in any other steps of this study) to express their ideas about the importance of each item. In order to assess the qulitatively content validity, 15 experts (three experts in clinical psychology [Shahid Beheshti University of Medical Sciences, Shahed University of Medical Sciences], four psychiatric nurses [University of Medical Sciences, Iran University of Medical Sciences], four nursing ethic trainers [Shahid Beheshti University of Medical Sciences, Tarbait Modarres University], and four nursing assistant professors, experts in development of relevant instruments [Shahid Beheshti University of Medical Sciences, Tehran University of Medical Sciences, Baghiatallah University of Medical Sciences, Isfahan University of Medical Sciences] were asked to review grammar, wording, item allocation, and scaling of the translated instrument. The comments were assessed, and one of the items was revised. The validated instrument was finalized and prepared to undergo the next phase. Further, we computed the content validation ratio (CVR) and content validity index (CVI) for this scale (26, 27). For measuring CVR, 15 experts assessed the questionnaire based on necessity. For measuring CVI, 15 experts assessed the questionnaire based on relevance.

\subsection{Factor analysis}

In factor analysis, the number of subjects needed is usually assessed in relation to the number of variables being measured. Although factor analysis is especially suitable when working with a large amount of data, the number of variables that may be included in a factor analysis procedure is limited. It is related to sample size. Certainly, the number of cases should always overstep the number of variables. A ratio of at least 10 subjects for each variable is favourable to generalize from the sample to a wider population (28). Therefore, 190 nurses were randomly selected by quota sampling. Participants were employees in critical care units affiliated with one major university of medical sciences in Tehran. As Severinsson reported, "Translation of questionnaires for cross-cultural research is needed"; methodological problems for validity may happen, for this reason an exploratory factor analysis (EFA) is essential 
(29). Confirmatory factor analysis (CFA) is a structural equation modelling (SEM) method, which is used to determine the goodness of fit between a hypothesized model and the data obtain from study cases. There are some fit indicators for deciding the goodness of fit of the model, and it is proposed that several indicators be considered $(30,31)$. In his study, indices of fit chi-square, root mean error of approximation (RMSEA), comparative fit index (CFI), and normed fit index (NFI) were measured. One of the common used indexes is chi-square. Because this index is affiliate with the sample size, the ratio of chi-square to the degree of freedom was used where values of 2-3 indicate an appropriate fit of the model. Another important index is the RMSEA, where values less than 0.08 are considered acceptable and less than 0.05 indicate a good fit of the model (31). Convenient values are $>0.9$ for CFI and NFI (32). CFA showed that the unidimensional model was not a good fit; therefore, EFA was done to explore a favorite structural model. EFA offered a new model based on five factors. We performed EFA through principal components analysis followed by a varimax rotation to test the factor constructs of all the 19 items for the ECNQCCV. Kaiser-Meyer-Olkin (KMO) test and Bartlett's test of sphericity were also evaluated to confirm sample adequacy for elicitation of the factors.

\subsection{Reliability}

The internal consistency was measured twice through Cronbach's alpha: first before and next after factor analysis. For calculating Cronbach's alpha, mean scores of 190 questionnaires were used. Then the reliability was measured by test-retest with a two-week interval on 30 nurses in critical care units with at least one-year experience.

\subsection{Ethical considerations}

The aim of study was explained to the participants by the first author. Research ethics approval was obtained from Shahid Beheshti Medical Sciences University Research Ethics Board in Tehran. Participants were asked to sign a consent form and were informed that they could withdraw from the study at any time. Then, both demographics (age, sex, educational level, marital status, and occupational characteristics) and the ECNQ-CCV questionnaires were given to the participating nurses to complete. Documented informed consent was obtained. The participants were asked to answer voluntarily and anonymously. The project was approved by the Research Ethics Committee of Shahid Beheshti University of Medical Sciences; also ethical authorization and agreement were obtained from the Research and Ethics Committees of University of Medical Sciences (ref. no.: IR.SBMU.PHNM.1394.190). Written informed consent was also acquired from all the participants who took part in the study.

\subsection{Data collection and analysis}

Collecting data was performed from April until June 2016. We analyzed the data using the IBM৫ SPSS $\odot$ Statistics version 20 (IBMC Corp., Armonk, NY, USA) and AMOS version 20.

\section{Results}

A total of 190 questionnaires were analyzed (response rate $=95 \%$ ). Women accounted for $72.1 \%$ of the population, and the mean age (SD) was 31.7 (5.6) years. Most of the participants (70.9\%) had a BSc in nursing (Table 1).

Table 1. Demographic and work characteristics of nurses $(n=190)$

\begin{tabular}{|l|l|l|l|}
\hline Variable & $n$ & $\%$ \\
\hline Gender & Female & 138 & 72.6 \\
\cline { 2 - 4 } & Male & 52 & 27.4 \\
\hline \multirow{3}{*}{ Educational level } & BS & 146 & 76.8 \\
\cline { 2 - 4 } & MSc & 22 & 11.6 \\
\hline Marital status & Single & 95 & 50 \\
\cline { 2 - 4 } & Married & 95 & 50 \\
\hline \multirow{3}{*}{ Organizational post } & General nurse & 134 & 70.5 \\
\cline { 2 - 4 } & In charge nurse & 50 & 26.3 \\
\cline { 2 - 4 } & Head nurse & 6 & 3.2 \\
\hline Working shift & Mornings & 28 & 14.7 \\
\cline { 2 - 4 } & Evenings & 32 & 16.8 \\
\cline { 2 - 4 } & Nights & 35 & 18.5 \\
\cline { 2 - 4 } & Rotation & 95 & 50 \\
\hline
\end{tabular}


Findings showed acceptable values ICVR $\geq 0.80, \mathrm{ICVI} \geq 0.78$, scale CVR $=0.96$, and its scale CVI $=0.92(\mathrm{Table}$ 2). Regarding factor analysis: data was tested by the Bartlett' s test of sphericity to achieve sample sufficiency (Bartlett' $\mathrm{s}$ test of sphericity; $\mathrm{p}<0.000, \mathrm{X}^{2}=2123.53$, and $\mathrm{KMO}=0.86$ ).

Table 2. Content validation ratio (CVR) and content validity index (CVI) for ECNQ-CCV_Persian language.

\begin{tabular}{|c|c|c|}
\hline CVI & CVR & \multirow[t]{2}{*}{ Scenario } \\
\hline Relevance & Necessity & \\
\hline 0.93 & 0.85 & $\begin{array}{l}\text { Scenario 1: Running treatments or performing tests, which are unnecessary in my } \\
\text { opinion because they lead us to an irreversible and terminal process }\end{array}$ \\
\hline 0.97 & 0.73 & $\begin{array}{l}\text { Scenario 2: Having to administer treatments or carry out procedures without the } \\
\text { permission of the critical patient, who is conscious but he/she do not knowing the } \\
\text { treatments purpose or the risks involved }\end{array}$ \\
\hline 0.94 & 1 & $\begin{array}{l}\text { Scenario 3: Caring for a patient who I believe should be on an ordinary hospital setting } \\
\text { rather than in the ICU }\end{array}$ \\
\hline 0.89 & 0.86 & $\begin{array}{l}\text { Scenario 4: Performing interventions or health care that gives institutional benefits more } \\
\text { than what they give to the patient }\end{array}$ \\
\hline 0.97 & 1 & $\begin{array}{l}\text { Scenario 5: Failure to keep patient's confidential clinical data by sharing them with third } \\
\text { parties or with people who are not directly involved in the patient's care }\end{array}$ \\
\hline 0.89 & 1 & $\begin{array}{l}\text { Scenario 6: Perform treatments and interventions without patient's families knowing the } \\
\text { objectives, benefits, and risks associated (when the patient has consented to the family } \\
\text { being informed) }\end{array}$ \\
\hline 0.97 & 0.9 & $\begin{array}{l}\text { Scenario 7: Realizing that the pain medication given to patients is not effective enough, } \\
\text { and the patient is suffering from pain }\end{array}$ \\
\hline 0.97 & 1 & $\begin{array}{l}\text { Scenario 8: Use of human or technical resources despite no improvement in clinical } \\
\text { status of patients in intensive care }\end{array}$ \\
\hline 0.93 & 1 & Scenario 9: Working with medical staff who I suspect are professionally incompetent \\
\hline 0.97 & 0.9 & $\begin{array}{l}\text { Scenario 10: Perform treatments and/or do interventions in accordance with the patient's } \\
\text { families wishes, despite knowing that are incompatible with the interests of the patient }\end{array}$ \\
\hline 0.97 & 0.85 & $\begin{array}{l}\text { Scenario 11: Perform treatments or applying procedures that are aggressive regarding } \\
\text { patient's situation and would cause more suffering in the patient }\end{array}$ \\
\hline 0.89 & 1 & $\begin{array}{l}\text { Scenario } 12 \text { : Working with nurses and nursing team that I consider to be professionally } \\
\text { incompetent }\end{array}$ \\
\hline 0.93 & 1 & $\begin{array}{l}\text { Scenario 13: Acting against my moral beliefs because there is not enough time for } \\
\text { appropriate care of the patient }\end{array}$ \\
\hline 0.97 & 1 & $\begin{array}{l}\text { Scenario 14: Performing within the framework of a research project or clinical trial } \\
\text { without as a nurse, being given all the information I consider necessary to carry out this } \\
\text { task }\end{array}$ \\
\hline 0.93 & 0.85 & $\begin{array}{l}\text { Scenario 15: Finding that is difficult to give timely information to the patient and his/her } \\
\text { family because the medical team discourages nurses from taking the initiative in this } \\
\text { regard }\end{array}$ \\
\hline 0.97 & 0.9 & $\begin{array}{l}\text { Scenario 16: Caring for a patient without knowing her or his opinion or option about } \\
\text { what treatments would be performed for keep her or him alive }\end{array}$ \\
\hline 0.89 & 1 & $\begin{array}{l}\text { Scenario 17: Perform treatments and procedures without, as a nurse, having been } \\
\text { previously involved in the decision to do so }\end{array}$ \\
\hline 0.97 & 1 & $\begin{array}{l}\text { Scenario 18: Failure to observe proper patient privacy during diagnostic procedures or } \\
\text { tests }\end{array}$ \\
\hline 1 & 1 & $\begin{array}{l}\text { Scenario 19: Lacking the equipment (space) or resources (time) that would enable the } \\
\text { clinical team to consider the ethical problems they have to deal with }\end{array}$ \\
\hline
\end{tabular}

Explorative factor analyses (EFA) is recognized five-factor model. When testing the factor pattern of all the 19 ECNQ-CCV items, five items were loaded on the first factor, six items were loaded on the second factor, four items were loaded on the third factor, two items were loaded on the forth factor, two items were loaded on the fifth factor (Table 3). However, some of the scenarios were in a factor not compatible with each other; thus, choosing the fit name for factors was not possible, and internal consistency of three of factors was low $(\alpha<0.7)$. The findings showed the fit model with one factor in confirmatory factor analysis (CFA) (Table 4, Figure 1). The coefficient of 
Cronbach's alpha for ECNQ-CCV before factor analysis was 0.74 and after that 0.92 . The intra-class coefficient correlation for test-retest reliability for ECNQ-CCV was 0.99 and $0.94(\mathrm{p}=0.000)$, respectively.

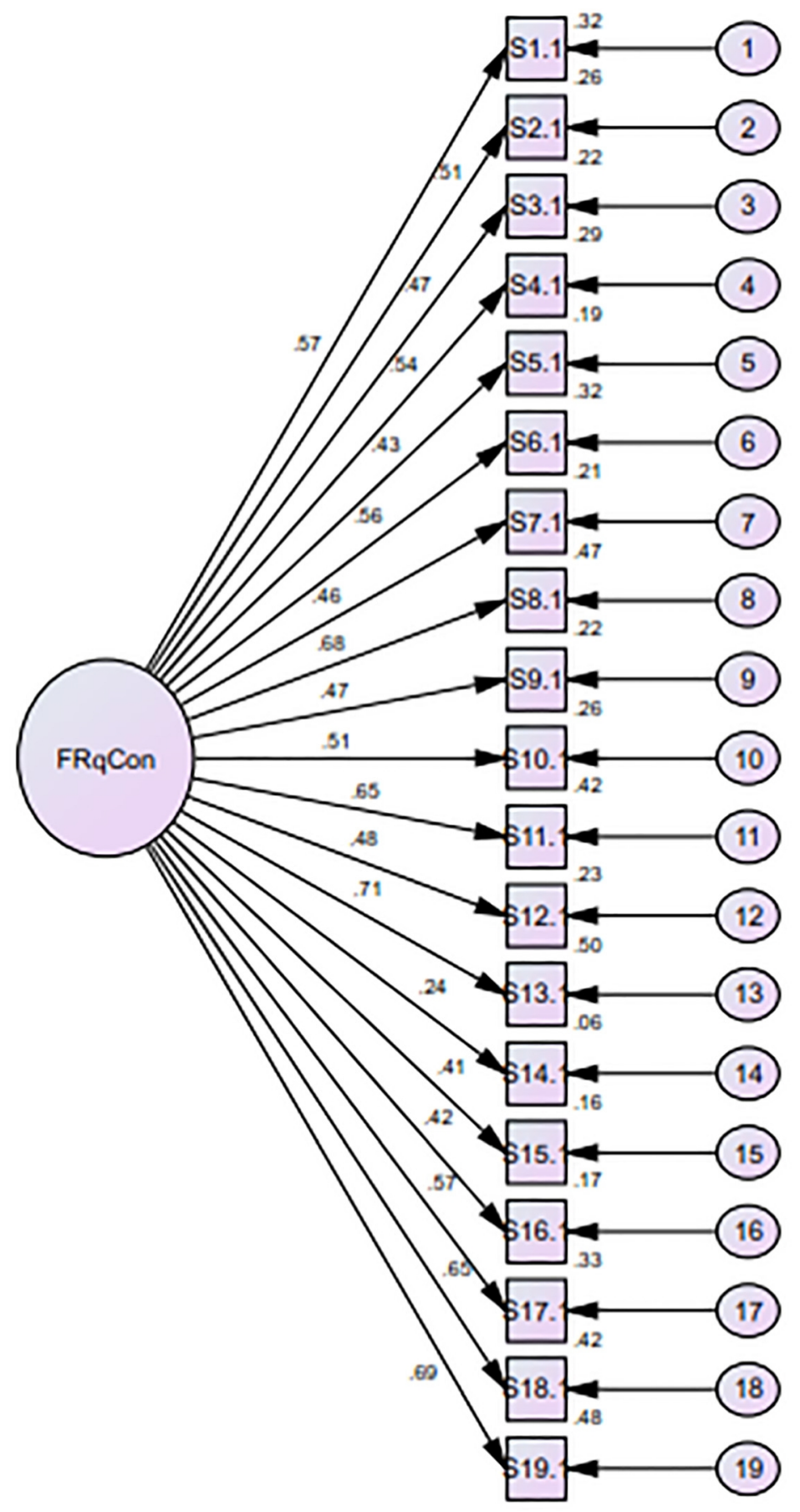

Figure 1. Confirmatory Factor Analysis for ECNQ-CCV-Persian Language 
http://www.ephysician.ir

Table 3. Rotated component matrix for ECNQ-CCV (Persian version)

\begin{tabular}{|l|l|l|l|l|l|}
\hline \multicolumn{5}{|l|}{ Rotated Component Matrix ${ }^{\mathrm{a}}$} \\
\hline & \multicolumn{3}{l|}{ Component } & \multicolumn{4}{l|}{} \\
\hline & 1 & 2 & 3 & 4 & 5 \\
\hline Scenario 10 & 0.622 & & & & \\
\hline Scenario 13 & 0.602 & & & & \\
\hline Scenario 17 & 0.659 & & & & \\
\hline Scenario 18 & 0.859 & & & & \\
\hline Scenario 19 & 0.760 & & & & \\
\hline Scenario 1 & & 0.766 & & & \\
\hline Scenario 2 & & 0.631 & & & \\
\hline Scenario 3 & & 0.590 & & & \\
\hline Scenario 8 & & 0.552 & & & \\
\hline Scenario 9 & & 0.635 & & & \\
\hline Scenario 12 & & 0.738 & & & \\
\hline Scenario 4 & & & 0.535 & & \\
\hline Scenario 14 & & & 0.782 & & \\
\hline Scenario 15 & & & 0.814 & & \\
\hline Scenario 16 & & & 0.532 & & \\
\hline Scenario 5 & & & & 0.824 & \\
\hline Scenario 6 & & & & 0.802 & \\
\hline Scenario 7 & & & & & 0.723 \\
\hline Scenario 11 & & & & & 0.663 \\
\hline
\end{tabular}

Table 4. Results of the confirmatory factor analysis of one-factor model of ECNQ-CCV

\begin{tabular}{|l|l|l|l|l|}
\hline & $\mathrm{X}^{2}(\mathrm{df})$ & $\mathrm{NFI}^{\mathrm{a}}$ & $\mathrm{CFI}^{\mathrm{b}}$ & RMSEA $^{\mathrm{c}}$ \\
\hline $\begin{array}{l}\text { One-factor } \\
\text { Hypothesis (ECNQ-CCV) }\end{array}$ & $625.42(152)$ & 0.57 & 0.63 & 0.12 \\
\hline
\end{tabular}

${ }^{a}$ Normed fit index. ${ }^{b}$ Comparative fit index. ${ }^{c}$ Root mean error of approximation. Sample $(n=190)$

\section{Discussion}

Using a valid and reliable instrument in any research is important. Invalid and unreliable instruments may result in incorrect or doubtful findings (33). Translation is a process through which a message in the source language can be transmitted to the target language. The basic issue in using a foreign questionnaire is translation and proper implementation of the research community's culture. Foreign questionnaires consistent with the culture of the target population provides the ability to compare results with other countries (34). The ECNQ-CCV was translated as well as adapted to Iranian culture and has good reliability and validity. The qualitative findings of this study during the translation process, content, and face validity were reported good and appropriate. The findings obtained from factor analysis showed one factor as an original instrument. In this study, none of scenarios was excluded, but, in another study, 12 scenarios were omitted (35). In Jahandar et al.'s study, the translation/adaptation process was not completed. But in this study, transcultural translation and validation of ECNQ-CCV was done completely. Of course, some of original scenarios were modified based on Iranian culture. In this research, the alpha value is good $(\alpha>0.70)$ but lower than in Jahandar et al.'s study (35) and Falcó-Pegueroles studies (2, 3, 8). This may be due to variation of population in the current study. Unfortunately, validation of ECNQ-CCV in another language was not completed due to the new instrument. While Thorne used two different measures of ethical conflict (ethical value congruence and shared ethical priorities) in her study, the first instrument has eight items that demonstrate high internal consistency $(\alpha=0.94)$, and the second instrument with five items demonstrated high internal consistency ( $\alpha=$ 0.84) (5). The "ethical conflict with hospitals" questionnaire designed by Guadine and Thorne showed good internal consistency $(\alpha>0.70)(1)$. In this study, one factor was revealed. This finding is consistent with the original version (2) but is different from that in Guadine and Thorne's study. Guadine and Thorne's study showed three factors (1). This could be due to differences in the instruments used. The Persian version of ECNQ-CCV has some differences from the original Spanish version of the tool. The part presented an alpha value lower than the original version (2) and other similar questionnaires (20-22). By the other part, CFA, one may deduce that the initial one dimensional hypothetical model does not offer a good fit to the data; thus, it is necessary to further study the dimensions in Persian language countries. However, the adapted questionnaire is a valid and useful tool for measuring conflict 
among Persian nurses. The sociodemographic characteristics are in the same line as that of previous similar studies $(3,21)$; however, in the Iranian nurse sample, there is a high proportion of male nurses. Because of the importance of the concept of ethical conflict in critical care nursing and its effect on the decision-making and the quality of nursing care, the importance of studying this aspect is undisputable. Professional nurses continuously compete in order to remain up-to-date to present the highest quality of patient care. Nevertheless, many factors have an impact on the quality of health care such as their health and work ability (36). Recent trends in health care have produced the potential for high levels of ethical conflict, particularly for physicians and nurses, who are key participants in the provision of quality ethical care (4). Ethical conflicts ordinarily begin as moral disagreements about an issue, the perception of injustice in the process of dealing with the issue, or an emotional response to a condition (37). They produce staff burnout and increase health care costs (38). Conflicts involving ethical, moral, and religious beliefs can be sources of anxiety, stress, and burnout for all those involved. More consideration is needed at a systems level to support a culture aimed at avoiding and reducing conflicts (17).

\section{Study limitations}

The limitations of this study are as follows: First, the sample size was small, which can result in the decrease of the statistical power of the study. Second, there is no similar study about validation of ECNQ-CCV in any language; thus discussion is difficult for researchers.

\section{Conclusions}

In sum, the results of the present study suggest that the Persian version of ECNQ-CCV, in addition to the appropriate validity and reliability, has affirmed construct validity in the one-factor model. This instrument can be used by critical care nurses and health care managers to identify and reduce the risk of ethical conflict and its personal, professional, and institutional consequences and, eventually, would help to improve the quality of patient care. Furthermore, ECNQ-CCV can be completed in a short time because of its simplicity and the small number of items.

\section{Acknowledgments:}

This study is part of a nursing master's dissertation and a research, approved by Shahid Beheshti University of Medical Sciences, Tehran, Iran. The present research was funded by the Shahid Beheshti University of Medical Sciences, Tehran, Iran. This article has been derived from a research project (Grant no.: 1394-7-29). The authors wish to thank all the participants and the members of expert committee.

\section{Conflict of Interest:}

There is no conflict of interest to be declared.

\section{Authors' contributions:}

All authors contributed to this project and article equally. All authors read and approved the final manuscript.

\section{References:}

1) Gaudine A, Thorne L. Nurses' ethical conflict with hospitals: A longitudinal study of outcomes. Nurs Ethics. 2012; 19(6): 727-37. doi: 10.1177/0969733011421626. PMID: 22619238.

2) Falcó-Pegueroles A, Lluch-Canut T, Guàrdia-Olmos J. Development process and initial validation of the ethical conflict in nursing questionnaire-critical care version. BMC Med Ethics. 2013; 14: 22. doi: 10.1186/1472-6939-14-22. PMID: 23725477, PMCID: PMC3711987.

3) Falcó-Pegueroles A, Lluch-Canut T, Roldan-Merino J, Goberna-Tricas J, Guàrdia-Olmos J. Ethical conflict in critical care nursing Correlation between exposure and types. Nurs Ethics. 2015; 22(5): 594-607. doi: 10.1177/0969733014549883. PMID: 25335920.

4) Gaudine A, LeFort SM, Lamb M, Thorne L. Clinical ethical conflicts of nurses and physicians. Nurs Ethics. 2011; 18(1): 9-19. doi: 10.1177/0969733010385532. PMID: 21285193.

5) Thorne L. The association between ethical conflict and adverse outcomes. Journal of Business Ethics. 2010; 92(2): 269-76. doi: 10.1007/s10551-009-0153-6.

6) Nurses AAoC-C. The 4 A's to rise above moral distress. Aliso Viejo, CA: American Association of Critical-Care Nurses; 2004.

7) Huffman DM, Rittenmeyer L. How professional nurses working in hospital environments experience moral distress: a systematic review. Crit Care Nurs Clin North Am. 2012; 24(1): 91-100. doi: 10.1016/j.ccell.2012.01.004. PMID: 22405714. 
8) Falcó-Pegueroles A, Lluch-Canut MT, Martínez-Estalella G, Zabalegui-Yarnoz A, Delgado-Hito P, ViaClavero G, et al. Levels of exposure to ethical conflict in the ICU: Correlation between sociodemographic variables and the clinical environment. Intensive Crit Care Nurs. 2016; 33: 12-20. doi: 10.1016/j.iccn.2015.10.004. PMID: 26796289.

9) Mareš J. Moral distress: Terminology, theories and models. Kontakt. 2016; 18(3): e137-e44. doi: 10.1016/j.kontakt.2016.07.001.

10) Henrich NJ, Dodek PM, Alden L, Keenan SP, Reynolds S, Rodney P. Causes of moral distress in the intensive care unit: A qualitative study. J Crit Care. 2016; 35: 57-62. doi: 10.1016/j.jcrc.2016.04.033. PMID: 27481736.

11) Cavaliere TA, Daly B, Dowling D, Montgomery K. Moral distress in neonatal intensive care unit RNs. Adv Neonatal Care. 2010; 10(3): 145-56. doi: 10.1097/ANC.0b013e3181dd6c48. PMID: 20505425.

12) Eizenberg MM, Desivilya HS, Hirschfeld MJ. Moral distress questionnaire for clinical nurses: instrument development. J Adv Nurs. 2009; 65(4): 885-92. doi: 10.1111/j.1365-2648.2008.04945.x. PMID: 19243462.

13) Shoorideh FA, Ashktorab T, Yaghmaei F, Majd HA. Relationship between ICU nurses' moral distress with burnout and anticipated turnover. Nurs Ethics. 2015; 22(1): 64-76. doi: 10.1177/0969733014534874. PMID: 24948793.

14) Atashzadeh Shorideh F, Ashktorab T, Yaghmaei F. Iranian intensive care unit nurses' moral distress A content analysis. Nurs Ethics. 2012; 19(4): 464-78. doi: 10.1177/0969733012437988. PMID: 22691602.

15) van Schijndel RJS, Burchardi H. Bench-to-bedside review: Leadership and conflict management in the intensive care unit. Crit Care. 2007; 11(6): 234. doi: 10.1186/cc6108. PMID: 18086322, PMCID: PMC2246194.

16) Azoulay E, Timsit JF, Sprung CL, Soares M, Rusinová K, Lafabrie A, et al. Prevalence and factors of intensive care unit conflicts: the conflicus study. Am J Respir Crit Care Med. 2009; 180(9): 853-60. doi: 10.1164/rccm.200810-1614OC. PMID: 19644049.

17) Danjoux Meth N, Lawless B, Hawryluck L. Conflicts in the ICU: perspectives of administrators and clinicians. Intensive Care Med. 2009; 35(12): 2068-77. doi: 10.1007/s00134-009-1639-5. PMID: 19756499.

18) Włodarczy D, Lazarewicz M. Frequency and burden with ethical conflicts and burnout in nurses. Nurs Ethics. 2011; 18(6): 847-61. doi: 10.1177/0969733011408053. PMID: 21974943.

19) Almost J. Conflict within nursing work environments: concept analysis. J Adv Nurs. 2006; 53(4): $444-53$. doi: 10.1111/j.1365-2648.2006.03738.x. PMID: 16448487.

20) Corley MC, Elswick RK, Gorman M, Clor T. Development and evaluation of a moral distress scale. J Adv Nurs. 2001; 33(2): 250-6. doi: 10.1046/j.1365-2648.2001.01658.x. PMID: 11168709.

21) Sporrong SK, Höglund AT, Arnetz B. Measuring Moral Distress in Pharmacy and Clinical Practice. Nurs Ethics. 2006; 13(4): 416-27. doi: 10.1191/0969733006ne880oa. PMID: 16838572.

22) Glasberg AL, Eriksson S, Dahlqvist V, Lindahl E, Strandberg G, Söderberg A, et al. Development and initial validation of the Stress of Conscience Questionnaire. Nurs Ethics. 2006; 13(6): 633-48. doi: 10.1177/0969733006069698. PMID: 17193804.

23) Jameton A. Nursing practice: The ethical issues. Prentice-Hall Englewood Cliffs. 1985; 22(4): 343. doi: 10.1016/0020-7489(85)90057-4.

24) LoBiondo-Wood G, Haber J. Nursing research: Methods and critical appraisal for evidence-based practice: Elsevier Health Sciences; 2014.

25) Polit DFY, Erances M. Measurement and the Measurement of Change. Netherlands: Wolters Kluwer; 2015.

26) Polit DF, Beck CT. Essentials of nursing research: Appraising evidence for nursing practice: Lippincott Williams \& Wilkins; 2013.

27) Polit DF, Beck CT, Owen SV. Is the CVI an acceptable indicator of content validity? Appraisal and recommendations. Res Nurs Health. 2007; 30(4): 459-67. doi: 10.1002/nur.20199. PMID: 17654487.

28) Munro BH. Statistical methods for health care research: Lippincott Williams \& Wilkins; 2005.

29) Severinsson E. Evaluation of the Manchester clinical supervision scale: Norwegian and Swedish versions. J Nurs Manag. 2012; 20(1): 81-9. doi: 10.1111/j.1365-2834.2011.01297.x. PMID: 22229904.

30) Brown TA. Confirmatory factor analysis for applied research. New York: Guilford Publications; 2015.

31) Seo DC, Torabi MR, Blair EH, Ellis NT. A cross-validation of safety climate scale using confirmatory factor analytic approach. J Safety Res. 2004; 35(4): 427-45. doi: 10.1016/j.jsr.2004.04.006. PMID: 15474546. 
32) Helsen K, Van den Bussche E, Vlaeyen JW, Goubert L. Confirmatory factor analysis of the Dutch Intolerance of Uncertainty Scale: Comparison of the full and short version. J Behav Ther Exp Psychiatry. 2013; 44(1): 21-9. doi: 10.1016/j.jbtep.2012.07.004. PMID: 22842528.

33) Atashzadeh-Shoorideh F, Yaghmaei F. Development and Psychometric Evaluation of Scales: A Survey of Published Articles. Journal of Medical Education. 2016; 14(4): 174-205.

34) Chrostowski SJ, Malak B. Translation and cultural adaptation of the TIMSS 2003 instruments. Boston: TIMSS \& PIRLS International Study Center, Lunch School of Education, Boston College. 2003; 93-108.

35) Jahandar F, Mohtashami J, Atashzadeh-Shorideh F, Pishgooie AH. Effectiveness of the negotiating style on ICU nurses' moral conflict in selected hospitals of Guilan University of Medical Sciences. Iranian Journal of Medical Ethics and History of Medicine. 2016; 9(1): 37-49.

36) Knezevic B, Milosevic M, Golubic R, Belosevic L, Russo A, Mustajbegovic J. Work-related stress and work ability among Croatian university hospital midwives. Midwifery. 2011; 27(2): 146-53. doi: 10.1016/j.midw.2009.04.002. PMID: 19589631.

37) Edelstein LM, DeRenzo EG, Waetzig E, Zelizer C, Mokwunye NO. Communication and conflict management training for clinical bioethics committees. HEC Forum. 2009; 21(4): 341-9. doi: 10.1007/s10730-009-9116-7. PMID: 19856111.

38) Fassier T, Azoulay E. Conflicts and communication gaps in the intensive care unit. Curr Opin Crit Care. 2010; 16(6): 654-65. doi: 10.1097/MCC.0b013e32834044f0. PMID: 20930623. 